\title{
ANÁLISIS DE LAS ESTRATEGIAS DE EDUCACIÓNY COMUNICACIÓN IMPLEMENTADAS POR ENTIDADES FINANCIERAS EN PEREIRA, ORIENTADAS A JÓVENES UNIVERSITARIOS ENTRE 18 Y 22 AÑOS
}

Education and communication strategies analysis implemented by financial entities in Pereira for young academics between 18 and 22 years

KARINA AGUDELO MEJÍA

Kagudelo5@estudiantes.areandina.edu.co

CRISTIAN CAMILO SÁNCHEZ

PAOLA ANDREA SERNA OSORIO (Docente investigadora)

Semillero de Investigación Nómadas laboratorio de tendencias Fundación Universitaria del Área Andina (Colombia)

Artículo de investigación formativa

Recepción: 3 de noviembre de 2020

Aceptación: 11 de marzo de 2021 


\title{
Resumen:
}

Este artículo presenta los resultados de una encuesta elaborada con los estudiantes de la institución de educación superior Fundación Universitaria del Área Andina, del programa de mercadeo y publicidad. La característica principal de esta investigación se basa en el análisis de la educación financiera y las estrategias de comunicación que tienen las entidades bancarias del sector de Pereira. A lo largo del tiempo se observó como principal factor del problema de las tarjetas de crédito, la facilidad para adquirir el producto y la falta de información sobre el mismo. La conclusión es que los jóvenes no están capacitados al momento de adquirir este tipo de producto ya que apenas están comenzando una vida crediticia sin las bases fundamentales para un buen uso de ellas. Además, de acuerdo con su comportamiento y su entorno son Jóvenes que tiene una facilidad de convencimiento más fácil pues el mercado, la publicidad y las ofertas continuas de las entidades financieras y de créditos de consumo (sobre todo los aspectos intangibles) provocan que hasta el consumidor más concientizado no controle las variables de endeudamiento y su entorno apunte a un declive de su economía personal. Por lo tanto, es evidente la necesidad de una regulación y un cambio de hábitos en los consumidores al momento de enfrentarse al mercado de forma inteligente

Palabras clave: educación; estrategias; comunicación; financiero; análisis.

\begin{abstract}
:
This article presents a survey results elaborated with the higher education institution students from Fundación Universitaria del Área Andina, in the marketing and advertising program. The main characteristic of this research is based on the financial education and the communication strategies analysis that the Pereira's banking entities sector have. Along the time it observes the ease to acquire the product and the lack of information as the principal problem in credit cards. It concludes that the young are not trained in the moment of purchase this kind of product; it barely is beginning a credit life without the fundamental bases for a good use of this type of money. Besides, in accordance with their behavior and its environment, they are young that have the ease of convincing easier; the marketing, advertising and continuous offers from financial entities and consumer credits (especially intangibles aspects) induce that up to the most conscientious consumer does not control the indebtedness variables and its environmental point to a slope of their personal economy. Therefore, it evidences the need of a regulation and a habit changes in consumer at the moment of face with the market in an intelligent way.
\end{abstract}

Keywords: education; strategies; communication; financial; analysis. 


\section{Introducción}

La presente investigación se plantea de acuerdo con el análisis realizado a los jóvenes del municipio de Pereira, departamento de Risaralda, Colombia; quienes a partir de los 18 años de edad son clientes potenciales para realizar adquisiciones de tarjetas de crédito. Estos jóvenes tienen como prioridad el uso de las redes sociales. Así, las entidades financieras tienen como estrategia abarcar este segmento de mercado de una manera masiva para tener mayor cantidad de clientes.

La característica principal de este análisis se basa en la falta de educación financiera y las estrategias de comunicación que tienen las entidades bancarias del sector de Pereira. Se puede decir que los jóvenes entre 18 y 22 años no están capacitados al momento de adquirir este tipo de productos pues apenas están empezando una vida crediticia sin las bases fundamentales para un buen uso de ellas.

Para analizar esta problemática es necesario mencionar las causas y efectos ocasionados en el municipio de Pereira. Una de ellas (la más importante) es la falta de educación financiera desde la educación media y terminando esta etapa en el desempleo. Entonces, cuando un usuario de una entidad financiera realiza un mal uso de un producto llamado tarjeta de crédito, esta acción se verá reflejada a largo plazo con un reporte negativo ante las centrales de riesgo y la consecuencia es un reporte negativo en data crédito. En ocasiones estos jóvenes no cuentan con la información suficiente para prevenir este tipo de situaciones.

\section{Planteamiento del problema}

Este trabajo realiza un análisis sobre las estrategias de educación financiera y comunicación de las entidades bancarias en el municipio de Pereira. En específico, en el uso de las tarjetas de crédito orientadas a jóvenes universitarios de 18 a 22 años. Esto con el ánimo de descubrir las particularidades que se presentan.

Según la Superintendencia Financiera de Colombia en la encuesta nacional sobre comportamientos, actitudes y conocimientos financieros, existe un alto porcentaje de jóvenes con cierto nivel de endeudamiento, desorden financiero y por tanto reportes negativos en centrales de riesgo. Esto sucede debido a que no hay cultura del buen hábito de pago; así como por la desinformación que tienen las entidades bancarias que no permite tener claridad sobre las afectaciones que conlleva a futuro a incurrir en demoras y retrasos en los pagos.

La investigación realizada sobre el tema del mal uso de las tarjetas de crédito por parte de los jóvenes en el municipio de Pereira se basa en la alta demanda en colocación de tarjetas y la prioridad de los entes financieros por captar una mayor cantidad de usuarios (según ABA BS Survey es de un 58\%). Por tanto, los obliga a generar un mayor impacto en redes sociales 
mejorando así sus estrategias para tener una atención personalizada con estos jóvenes; además de tener una publicidad atractiva enmarcada en el ámbito nacional y local.

La importancia que tiene este tema es relevante en la actualidad debido a los desafíos a los cuales se va centrando la educación. Asimismo, las estrategias que se tiene para suplir este tipo de necesidades sobre la adquisición de productos financieros y la facilidad con la que se puede obtener este tipo de productos.

\section{Objetivo general.}

Analizar las estrategias de comunicación y educación implementadas por las entidades bancarias de Pereira para el uso de las tarjetas de crédito, orientadas a jóvenes universitarios de 18 a 22 años.

\section{Objetivos específicos.}

- Identificar las estrategias de comunicación y educación que las entidades bancarias realizan en Pereira, orientadas a jóvenes de 18 a 22 años.

- Evaluar los reportes en centrales de riesgos que presentan los jóvenes con edades entre 18 y 22 en el manejo de tarjetas de crédito en Pereira.

- Determinar los comportamientos y hábitos de consumo de tarjetas de crédito que tienen los jóvenes entre 18 y 22 años de Pereira.

\section{Marco teórico}

La tarjeta de crédito es un medio de pago que permite realizar compras y cancelar dicho valor posteriormente. Se denomina "crédito" porque el monto que se utiliza al realizar la compra corresponde a un préstamo que otorga la entidad financiera.

Al hacer uso de este producto, el banco o entidad financiera autoriza a hacer uso de cierto monto de dinero para pagar bienes o servicios. El comercio cobra el valor y luego el banco hará llegar un extracto. Allí se notificará el valor del costo o servicio que se adquirió; la tasa de interés que se cobrará y los costos asociados que esta operación tendría.

No obstante, los jóvenes al encontrar tanta facilidad al adquirirla, no miden las consecuencias de un mal uso. Estar reportado en una central de información financiera (también conocidas como centrales de riesgo) es una consecuencia de no pagar las deudas contraídas con las entidades bancarias e instituciones a tiempo.

\section{La comunicación de la entidad financiera con el mercado.}

Desde principios de la década de 1970 la estrategia de comunicación se ha convertido en uno de los puntos clave del marketing bancario. La mayor presencia y acción de la competencia, el desarrollo de nuevos productos 
y servicios, la necesidad de diferenciarse y posicionarse en determinados segmentos del mercado. Además, el empeño por rentabilizar la red de oficinas y, en general, las condiciones rápidamente cambiantes del mercado financiero, complejas y efectivas, son integradas en los correspondientes planes de marketing.

Esta necesidad ha llevado a las entidades financieras a invertir grandes sumas no solo en publicidad, sino también en otras estrategias de comunicación. Efectivamente, la acción de comunicar no se circunscribe solo a la publicidad (aunque esta continúe siendo muy importante). Sino que además se usan otras estrategias como son las relaciones públicas, las promociones y la venta personal.

Hay que tener en cuenta que los productos financieros son realmente servicios y como tales no pueden evaluarse físicamente a priori antes de su compra. Esto hace que para el presente caso la comunicación sea aún más importante; ya que básicamente es la única herramienta la cual dispone.

\section{Segmentación y comportamientos del consumidor.}

Los consumidores buscan mediante productos satisfacer sus necesidades no solo básicas y fisiológicas, también hedonistas. Aquellas que les permitan sentir experiencias y placer. Sin embargo, muchos de los productos que compra la gente no lo hacen por sus funciones, sino por lo que significan, es decir, los productos tienen un papel que va más allá de las tareas que desempeñan para las personas.

En este sentido, las motivaciones vienen de las diferentes necesidades del ser humano que conllevan a satisfacer primero las más básicas, para continuar a satisfacer las de seguridad, las sociales, las correspondientes a la autoestima y las de autorrealización. Cada necesidad se va satisfaciendo a su manera, empezando desde la base de la pirámide que son las necesidades fisiológicas, moviéndose hacia arriba por los deseos emocionales y finalmente las psicológicas.

Las diferentes formas de pago en jóvenes varían según sus comportamientos de consumo. De acuerdo con el segmento del mercado, menciona que los jóvenes en Colombia tienen un perfil similar de comportamiento en cuanto a la forma de consumo. El 35 \% de los encuestados eran estudiantes, utilizan la forma de pago para actividades generales como compras, comidas, viajes y entretenimiento. El 79\% utiliza efectivo, el 57\% asegura hacer uso de tarjetas de débito y $48 \%$ de tarjetas de crédito. Con un menor porcentaje, se encuentra el pago con cheques (7\%) y Monedero Electrónico (4\%). Así, se identifica una necesidad de adquirir productos y servicios financieros a temprana edad, sin conocimientos básicos de los costos que generan las tarjetas de crédito como: interés, cuota de manejo, seguros, costo del plástico, gravámenes financieros, entre otros. 
Existen muchos estudios sobre el comportamiento del consumidor en distintos contextos socioculturales. Particularmente, este tipo de estudios son contratados por empresas productoras - distribuidoras de bienes y servicios, a las cuales les interesa conocer la estructura de consumo de su población objetivo o nicho de mercado.

Sin embargo, existen organizaciones (instituciones sin ánimo de lucro, hospitales, escuelas e instituciones de educación superior) interesadas en conocer el comportamiento de los individuos que hacen parte de ellas. Un ámbito fundamental para conocer a los individuos es conocer sus hábitos de consumo, ligados a diferentes aspectos socioculturales y pueden caracterizar una población en general (Moreno y Rodríguez, 2005; Gutiérrez Quecano y Maldonado, 2019).

El comportamiento del consumidor se puede abordar desde distintas dimensiones. Abarca una gran cantidad de campos, entre ellos el estudio de los procesos que intervienen cuando una persona o grupo selecciona, adquiera, usa o desecha productos, servicios, ideas o experiencias para satisfacer necesidades y deseos.

Los consumidores poseen características muy distintas, dependiendo su tipo, edad o etnia. El consumidor siempre se desenvolverá en un mercado donde gran parte de la conducta parte de un proceso conocido como comportamiento de compra, reflejando una relación estrecha entre consumidores y productores. Esta relación se basa en el intercambio, en la que los dos (2) partícipes dan y reciben algo de valor (Solé, 2001).

Además de factores tales como edad, género, estructura familiar e ingresos, también influye la cultura popular. Influencia a consumidores en aspectos como la música, los libros, las películas, los deportes, las celebridades y otras formas de entretenimiento. Los llevan a consumir artículos que a primera vista no son necesarios. En la construcción de un esquema formal del comportamiento del consumidor, el objetivo es describir la elección de consumo. Allí donde sea evidente la preferencia por aquellos bienes que otorgan satisfacción o bienestar, condicionado al ingreso monetario que se posee (Díaz, 2014).

\section{Entidades financieras y tarjetas de crédito en Pereira}

Pereira cuenta con una gran variedad de entidades financieras; dentro de sus servicios ofrece tarjetas de crédito y un amplio portafolio. Se ubica el Grupo Aval (conformado por el banco de Bogotá, Av. Villas, Banco de Occidente y el Banco Popular). Además, Scotiabank-Colpatria, Bancolombia, Davivienda, Banco Caja Social, Banco Itaú, Banco Pichincha, Serfinansa, Banco Falabella, Banco BBVA, Banco Agrario y Bancoomeva.

Estos bancos ofrecen todo tipos de productos financieros. Principalmente, se enfocan en destacar las tarjetas de créditos por su facilidad de adquisición. En el Banco de Bogotá, los clientes entre 18 y 25 años pueden solicitar la tarjeta de crédito joven, con el ciento por ciento del cupo para retiros de efectivo en cajeros (Banco de Bogotá, s.f.). 
Asimismo Davivienda tiene la tarjeta de crédito 40 principales, dirigida a estudiantes de grado once y universitarios. Tiene un cupo de crédito rotativo (Davivienda, s.f.). Sin embargo, Bancolombia ofrece tarjetas de crédito joven, dirigida a jóvenes entre 14 y 25 años de edad, con un cupo de crédito rotativo en pesos desde \$200.000 hasta \$5.000.000. Lo pueden utilizar para realizar compras y avances de efectivo en Colombia y en el exterior (Bancolombia, s.f.).

No obstante, Banco de Occidente tiene la tarjeta de crédito credencial MasterCard Juvenil. Está diseñada para estudiantes de siete (7) a 30 años, dependientes económicamente y respaldados por un codeudor. Con disponibilidad del 100\% del cupo asignado para realizar transacciones de avance en efectivo (Banco de Occidente, s.f.).

\section{Tarjetas de crédito y su consumo en jóvenes.}

Según un artículo publicado por el periódico El Tiempo, afirma que los jóvenes entre 18 y 22 años son más activos en su vida crediticia que la generación anterior conocida como Millennials. Entre ellos, el producto bancario más popular es la tarjeta de crédito, junto con el crédito educativo. Los dos (2) se posicionan como lo más demandado entre esta juventud, según un nuevo estudio de la firma TransUnion.

En tarjetas de crédito, la participación de las mujeres en ha crecido en los últimos años. Llegó a 50,7\% mientras que en el 2017 era 49,7\%. Esto fue impulsado por las tarjetas de retail. Entonces, son 2,8 millones los jóvenes de esta generación entre los 18 y 22 años que tienen deuda en el sector financiero o real. Según el informe, si se incluye además obligaciones del sector real es más probable que tengan una obligación contraída con el sector de telecomunicaciones para la adquisición de aparatos tecnológicos o servicios (El Tiempo, 2020).

El problema del consumo ha arrojado interesantes hallazgos. Algunos estudios han relacionado el número de tarjetas de crédito que un individuo posee con la cantidad de dinero gastada, el número de compras o con la compra de productos más caros. Así, se ha encontrado una relación directa y positiva entre la deuda y el uso de tarjetas de crédito.

También se han realizado intentos por relacionar el endeudamiento con el nivel de ingresos. Es decir, una mayor deuda en sujetos que hacían uso irracional de las tarjetas tenía un número superior de cuentas corrientes y, sin embargo, poseían ingresos medios. Este fenómeno se debe a que las personas de estrato cinco (5) y seis (6) tienen una mayor capacidad financiera y pueden acceder a muchos más créditos, lo que les permite incluso sobregirarse.

Por su parte, las entidades financieras los ven como clientes preferenciales y les pueden ofrecer mucho más dinero que el que realmente podrían pagar o estar en el límite por el pago de las cuotas. En general, acceden a créditos 
en los meses de mayo y octubre, meses donde están próximos a recibir un ingreso extra, ya que se efectúa el pago de las primas (cantidad de dinero que el gobierno u otro organismo concede como incentivo por la consecución de ciertos objetivos o cumplimiento de ciertos requisitos). Especialmente, por la línea de crédito de consumo espera pagar parte de ese dinero con las primas.

\section{Diseño metodológico}

Esta investigación es de tipo documental informativa con profundidad de investigación descriptiva. Se basa en la revisión de diversas fuentes de información que van desde fuentes primarias, hasta textos que abordan temas existentes en relación con la investigación. La investigación documental se realiza a través de la consulta de documentos.

Esta investigación relaciona dos (2) categorías de análisis: educación financiera en jóvenes entre 18 y 22 años y las estrategias de comunicación que tienen las entidades financieras enfocadas a este segmento. Se realiza bajo un enfoque de investigación cuantitativa para comprender la manera como desde su educación los jóvenes entre 18 y 22 años hacen uso de las tarjetas de crédito. Ahora bien, en términos de estrategias y comunicación analizar la relación directa de la educación y la persuasión de estas entidades tienen con el mal uso que se le da a este producto.

\section{Muestra.}

La investigación se decidió aplicar solo a estudiantes universitarios del programa de mercadeo y publicidad de la Fundación Universitaria del Área Andina. Esta muestra se realizó con el método probabilístico con un muestreo por conglomerados. Allí los estudiantes son seleccionados al azar en forma natural por agrupaciones. Los grupos son heterogéneos para obtener resultados precisos. Además, esta muestra se realizó en el mes de agosto y octubre.

\section{Técnicas para la recolección de la información.}

La técnica de esta investigación se realiza mediante la aplicación de una encuesta cerrada a los estudiantes de mercadeo y publicidad de la Fundación Universitaria del Área Andina, con el fin de conocer estados de opinión y hechos específicos sobre el tema. La intención de la encuesta no es describir los individuos particulares quienes, por azar, son parte de la muestra; en cambio, obtener un perfil compuesto de la población. 


\section{Resultados preliminares y conclusiones}

Si se fomenta una educación financiera a la ciudadanía, se podrá contribuir y empoderar al consumidor financiero para que sean capaces de proteger sus derechos y exigir el cumplimiento de los deberes de las entidades financieras. En consecuencia, se promoverá la autorregulación de estas entidades. En otras palabras, con programas de educación financiera se tendrá personas informadas y mejor capacitadas para evaluar los servicios que reciben, los costos y riesgos que asumen.

Finalmente, hay que resaltar que en Colombia el dominio de conceptos financieros básicos aún no se ha definido como una competencia básica que debe tener cualquier profesional. Esta situación contradice la cultura de emprendimiento que ha sido apoyada por instituciones educativas. 


\section{Referencias bibliográficas}

Díaz, A. A. (2014). Oligopolio y poder de mercado. Discusión de la hipótesis de precios rígidos. Economía Informa, 384, 109-127.

El Tiempo. (7 de febrero de 2020). Tarjeta de crédito, el producto principal entre menores de 25 años. El Tiempo. Recuperado de https:// www.eltiempo.com/economia/ sectores/tarjetas-de-credito-elproducto-que-mas-usan-los-jovenes-menores-de-25-anos-459052.

Gutiérrez Quecano, R. y Maldonado Ladino, M. (2019). Elementos epistemológicos y socioculturales de la gastronomía. En Royer Gutiérrez. (Comp.) Tendencias en los procesos de formación del gastrónomo en Colombia (pp. 8-27). Bogotá: Editorial Universitaria San Mateo.

Moreno, L., y Rodríguez, G. (2005). Nutrición en la Adolescencia. Madrid: Grupo Acción Médica.

Solé, M. L. (2001). Los Consumidores del Sig/o XXI. Madrid: Escuela Superior de Gestión Comercial y Marketing (ESIC) (Ed. 2). 